\title{
Stability analysis for a thermodynamically consistent model of relativistic fluid dynamics
}

\author{
Laura Stricker and Hans Christian Öttinger \\ ETH Zürich, Department of Materials, Polymer Physics, CH-8093 Zurich, Switzerland
}

(Received 5 September 2018; published 10 January 2019)

\begin{abstract}
In relativistic fluid mechanics, positive entropy production is known to be insufficient for guaranteeing stability. Much stronger criteria for thermodynamic admissibility have become available in nonequilibrium thermodynamics. We here perform a linear stability analysis for a model of relativistic hydrodynamics that is based on the general equation for the nonequilibrium reversible-irreversible coupling (GENERIC) framework of nonequilibrium thermodynamics. Assuming a quadratic entropy function near equilibrium, we find stability for the entire range of physically meaningful model parameters for relativistic fluid dynamics based on GENERIC. The search for thermodynamic admissibility moreover reveals a fundamental difference between liquids and gases in relativistic fluid dynamics.
\end{abstract}

DOI: 10.1103/PhysRevE.99.013105

\section{INTRODUCTION}

Relativistic fluid dynamics plays an important role in astrophysics and cosmology as, for example, it allows us to describe the collapse of stars into neutron stars, flows around black holes, jets with relativistic speeds originating from the core of active galactic nuclei, the formation of galaxies, or the expansion of the entire universe. The first use of relativistic fluid dynamics in the field of high-energy physics appeared in the 1950s, in the works of Landau [1] and Khalatnikov [2]. In recent years, the topic received renewed attention due to its potential to describe experiments on quark-gluon plasmas produced in relativistic heavy ion colliders [3-6].

The first attempts to generalize the laws of fluid mechanics and thermodynamics to the relativistic context were based on an extended Fourier law for heat conduction [7,8]. These formulations suffered from two fundamental flaws, which are closely related to each other $[9,10]$ : they are unstable $[11,12]$, and the parabolic nature of the corresponding differential equation leads to instantaneous propagation of heat, hence violating causality [13]. To overcome this problem, some authors added an ad hoc relaxation term to Fourier's law, turning the parabolic equation into a hyperbolic one with finite propagation speed $[14,15]$. Later studies showed that instability is the consequence of an ill-posed initial value problem when using parabolic equations [16] and that parabolic equations can still be adopted if formulated in a well-posed manner [17]. Conversely, hyperbolic equations can result in well-posed problems with finite but superluminal propagation speed. Hence, considering the physically acceptable range of

Published by the American Physical Society under the terms of the Creative Commons Attribution 4.0 International license. Further distribution of this work must maintain attribution to the author(s) and the published article's title, journal citation, and DOI. parameters is essential in determining the stability and validity of a theory [17].

A big step forward in formulating relativistic fluid dynamics resulted from the insight that the entropy should depend on additional structural variables (related to the momentum and energy fluxes) and that the heat flux should not be proportional to the entropy flux. In 1967 Müller demonstrated in the context of heat flow that such a more complete description of nonequilibrium states solves the problem of infinite propagation speeds, and he moreover showed that such a description emerges naturally from kinetic gas theory [18]. It was then natural to extend Grad's moment method for the Boltzmann equation [19] to the relativistic context [20-23]. The derivation of moment equations from the relativistic Boltzmann equation culminated in what is now known as the IsraelStewart theory [24,25]. All work along these lines suffers from the infamous closure problem [26] for the moment hierarchy.

The problem of relativistic fluid dynamics has also been approached with the tools of nonequilibrium thermodynamics. The basic idea of extended irreversible thermodynamics (EIT) is to consider the dissipative fluxes (e.g., the heat flux vector and the viscous pressure tensor) as independent variables and to formulate convection-relaxation equations for such fluxes [27]. In the limit of slow phenomena, these equations reduce to the classical constitutive laws, but they are also suitable to describe fast phenomena, since they lead to hyperbolic equations with finite speeds of propagation for thermal and viscous perturbations. Such models are compatible with the kinetic theory of Grad's 14-moment method [28]; they are compatible with causality and stable under specific constraints [10]. The general equation for the nonequilibrium reversibleirreversible coupling (GENERIC) framework of nonequilibrium thermodynamics is more versatile in the choice of variables than EIT and more restrictive in the structure of the equations, in particular, by imposing a Hamiltonian structure on reversible dynamics. A GENERIC model of relativistic fluid dynamics has been developed in Refs. [29,30], and gravity has been included in Refs. [31,32]. Further possible 
approaches include Carter's theory [33], the Ván-Biró model [17], and a conformal field theory formulation [34].

In the present paper we study the linear stability of the GENERIC model developed in Refs. [29,30]. We first present these equations for relativistic fluid dynamics and highlight their most important features.

\section{MODEL}

We restrict ourselves to special relativity. For the metric, we adopt the Minkowski tensor with the convention $\left(\eta^{\mu \nu}\right)=$ $\operatorname{diag}(-1,1,1,1)$. For the indexes of four-vectors and tensors, we use Greek letters to denote components from 0 to 3 (i.e., time and space components) and Latin letters to denote components from 1 to 3 (i.e., space components only).

\section{A. Balance equations}

We introduce the dimensionless fluid velocity four-vector $u^{\mu}$ in such a way that we obtain the relativistic continuity equation

$$
\partial_{\mu}\left(\rho_{\mathrm{f}} u^{\mu}\right)=0
$$

where $\rho$ is the mass density and the subscript $f$ denotes quantities evaluated in the comoving local reference frame of the fluid. In terms of the components $v^{i}$ of the local fluid velocity $\boldsymbol{v}$, we have

$$
u^{0}=\gamma, \quad u^{i}=\gamma \frac{v^{i}}{c} \quad(i=1,2,3)
$$

where $c$ is the speed of light and $\gamma=\left(1-\boldsymbol{v}^{2} / c^{2}\right)^{-1 / 2}$ is the Lorentz factor, chosen in such a way that

$$
u^{\mu} u_{\mu}=-1 \text {. }
$$

The energy and momentum balances in the relativistic setting are combined and can be expressed as

$$
\partial_{\mu} T^{\mu \nu}=0,
$$

where $T_{\mu \nu}$ is the energy-momentum tensor. Such a tensor has to incorporate energy and momentum densities as well as energy and momentum fluxes.

In defining the local fluid velocity, different choices are possible: in the Eckart framework, the particle flow vanishes in the local rest frame of the fluid [7] whereas, in the Landau framework, the momentum density vanishes in the local rest frame [8]. As, according to the GENERIC framework (and also in the generalized bracket approach of Beris and Edwards [35]), Hamiltonian structure is postulated to be a hallmark of reversible dynamics, the introduction of the proper velocity to be used for the reversible convection mechanism is an important issue in these frameworks. The natural choice is to implement the continuity equation (1) through the definition of velocity and to determine the energy-momentum tensor in Eq. (4) from the requirement that the entropy is conserved under the reversible dynamics generated by any Hamiltonian, not just the physical one (see also footnote on p. 168 of Ref. [36]). The Hamiltonian structure of reversible dynamics and the conservation of entropy under reversible dynamics for any choice of the Hamiltonian are the powerful features of the GENERIC framework in formulating relativistic fluid dynamics.

\section{B. Relaxation equations}

In order to avoid the presence of spatial derivatives in the formulation of the momentum and energy fluxes, and in the spirit of the rheological modeling of complex fluids [36,37], we introduce the structural variables $\alpha_{\mu \nu}$ and $\omega_{\mu}$ (for a detailed motivation and derivation of all the equations, see Sec. 5.2 of the monograph [36] or Sec. 2 of the original paper [29]). The relation of these structural variables to the physical energy-momentum tensor and entropy flux emerges after specifying the convection mechanism and the entropy density in Eqs. (13) and (16) below.

The evolution equation for the four-vector $\omega_{\mu}$ is given by

$$
u^{\nu}\left(\partial_{\nu} \omega_{\mu}-\partial_{\mu} \omega_{\nu}\right)=-\frac{1}{c \lambda_{1}} \hat{\eta}_{\mu \nu} \omega^{\nu},
$$

where $\hat{\eta}^{\mu v}=\eta^{\mu v}+u^{\mu} u^{v}$ is the spatial projector and $\lambda_{1}$ is a characteristic relaxation time. The convection term on the left-hand side is dictated by its Hamiltonian nature. The relaxation term on the right-hand side is chosen to be linear for simplicity. The projector $\hat{\eta}^{\mu \nu}$ on the right-hand side needs to be introduced because the left-hand side vanishes after contraction with $u^{\mu}$. From now on, we write $\hat{\omega}_{\mu}=\hat{\eta}_{\mu \nu} \omega^{\nu}$ for the four-vector with the desired property

$$
u^{\mu} \hat{\omega}_{\mu}=0 .
$$

For $\boldsymbol{v}=\mathbf{0}$, Eq. (5) becomes

$$
\partial_{0} \omega_{j}=\partial_{j} \omega_{0}-\frac{1}{c \lambda_{1}} \omega_{j}
$$

so that $\omega_{j}$ relaxes to the temperature gradient, or the driving force for the heat flux, provided that $\omega_{0}$ is chosen as the temperature. More precisely, we impose the Lorentz invariant condition

$$
u^{\mu} \omega_{\mu}=T_{\mathrm{f}},
$$

where $T_{\mathrm{f}}$ is the temperature in the local rest frame.

Similarly to the equation for $\omega_{\mu}$, also the evolution equation for the symmetric four-tensor $\alpha_{\mu \nu}$ is given by the interplay of Hamiltonian convection and linear relaxation,

$$
u^{\lambda}\left(\partial_{\lambda} \alpha_{\mu \nu}-\partial_{\mu} \alpha_{\lambda \nu}-\partial_{\nu} \alpha_{\mu \lambda}\right)=-\frac{1}{c \lambda_{0}} \bar{\alpha}_{\mu \nu}-\frac{1}{c \lambda_{2}} \stackrel{\alpha}{\mu \nu}_{\mu},
$$

where $\lambda_{0}$ and $\lambda_{2}$ are the characteristic relaxation times for the independently relaxing trace-part $\bar{\alpha}_{\mu \nu}$ and trace-free part $\stackrel{\circ}{\alpha}_{\mu \nu}$ of $\alpha_{\mu \nu}$. To make both sides of Eq. (9) vanish upon contraction with $u^{\mu}$ or $u^{v}$, we choose

$$
\bar{\alpha}_{\mu \nu}=\frac{1}{3}\left(\alpha_{\lambda}^{\lambda}-1\right) \hat{\eta}_{\mu \nu}, \quad \stackrel{\circ}{\alpha}_{\mu \nu}=\alpha_{\mu \nu}+u_{\mu} u_{\nu}-\bar{\alpha}_{\mu \nu}
$$

and

$$
\alpha_{\mu \lambda} u^{\lambda}=u_{\mu} .
$$

For these conditions, $\alpha_{\mu \nu}$ relaxes to the symmetrized velocity gradient tensor, or to the driving force for the momentum flux [see the linearized Eq. (24) below]. Equation (11) implies that $\alpha_{\mu \nu}$ must be dimensionless, and, as a symmetric tensor, it has only six independent components. The simplicity and 
elegance of the convection terms on the left-hand sides of Eqs. (5) and (9) should be noted.

\section{Entropy balance}

The balance and relaxation equations of the preceding two sections feature a Hamiltonian convection mechanism. Another important feature of the GENERIC framework is the requirement that the entropy must be conserved under reversible dynamics for any choice of the Hamiltonian. In the rheological modeling of complex fluids, this requirement provides the stress tensor. In relativistic fluid dynamics, it provides the energy-momentum tensor. To proceed, we need to specify the entropy density $s_{\mathrm{f}}$ in the local rest frame.

We assume that $s_{\mathrm{f}}$ depends on the mass density $\rho_{\mathrm{f}}$ and internal energy density $\epsilon_{\mathrm{f}}$, as well as on the structural variables, i.e., it can be expressed as $s_{\mathrm{f}}\left(\rho_{\mathrm{f}}, \epsilon_{\mathrm{f}}, \alpha_{\mu \nu}, \hat{\omega}_{\mu}\right)$. The spatial projection $\hat{\omega}_{\mu}$ is used here instead of $\omega_{\mu}$ because the temporal part of $\omega_{\mu}$ includes thermodynamic information and would be redundant with $\rho_{\mathrm{f}}$ and $\epsilon_{\mathrm{f}}$. The entropy density is independent of the local fluid velocity. We can now provide a definition of the temperature $T_{\mathrm{f}}$ in Eq. (8) in terms of $s_{\mathrm{f}}\left(\rho_{\mathrm{f}}, \epsilon_{\mathrm{f}}, \alpha_{\mu \nu}, \hat{\omega}_{\mu}\right)$,

$$
\frac{1}{T_{\mathrm{f}}}=\frac{\partial s_{\mathrm{f}}}{\partial \epsilon_{\mathrm{f}}} \text {. }
$$

Equation (12) is formally similar to the classical definition of thermodynamic temperature, but it entails some additional ambiguity. In the equilibrium setting, the derivative of $s_{\mathrm{f}}$ with respect to $\epsilon_{\mathrm{f}}$ is performed keeping $\rho_{\mathrm{f}}$ constant. Here, in general, we additionally have to keep the structural variables constant. Therefore the definition of the temperature can depend on the particular choice of the structural variables.

According to the development of thermodynamically admissible equations for relativistic fluid dynamics in Sec. 5.2 of Ref. [36] or Sec. 2 of Ref. [29], the entropy balance can be written in the form (see the Appendix)

$$
\begin{aligned}
\partial_{\mu}\left(s_{\mathrm{f}} u^{\mu}+T_{\mathrm{f}} \frac{\partial s_{\mathrm{f}}}{\partial \hat{\omega}_{\mu}}\right)= & -\frac{\partial s_{\mathrm{f}}}{\partial \alpha_{\mu \nu}}\left(\frac{1}{c \lambda_{0}} \bar{\alpha}_{\mu \nu}+\frac{1}{c \lambda_{2}} \stackrel{\alpha}{\mu \nu}_{\mu \nu}\right) \\
& -\frac{1}{c \lambda_{1}} \frac{\partial s_{\mathrm{f}}}{\partial \hat{\omega}_{\mu}} \hat{\omega}_{\mu} .
\end{aligned}
$$

The nonconvective contribution to the entropy-flux fourvector is given by $T_{\mathrm{f}} \partial s_{\mathrm{f}} / \partial \hat{\omega}_{\mu}$, so that we have a clear relationship between the entropy flux and the spatial part of the structural variable $\omega_{\mu}$. According to Eq. (8), the temporal part of $\omega_{\mu}$ is given by the temperature.

A nonnegative entropy production on the right-hand side of Eq. (13) can be achieved either by requiring $\partial s_{\mathrm{f}} / \partial \alpha_{\mu \nu}$ and $\partial s_{\mathrm{f}} / \partial \hat{\omega}_{\mu}$ to be negative multiples of $\alpha^{\mu \nu}$ and $\hat{\omega}^{\mu}$, respectively, or by replacing the linear relaxation mechanism by one that is driven by nonlinear entropy derivatives. Whereas the latter option is much more general, we here insist on a linear relaxation term and use the quadratic entropy density

$$
\begin{aligned}
& s_{\mathrm{f}}\left(\rho_{\mathrm{f}}, \epsilon_{\mathrm{f}}, \alpha_{\mu \nu}, \hat{\omega}_{\mu}\right) \\
& \quad=s_{\mathrm{f}}^{\mathrm{eq}}\left(\rho_{\mathrm{f}}, \epsilon_{\mathrm{f}}\right)-\frac{1}{2} \rho_{\mathrm{f}}\left[H_{\alpha}\left(\alpha_{\mu \nu} \alpha^{\mu \nu}-1\right)+H_{\omega} \hat{\omega}_{\mu} \hat{\omega}^{\mu}\right],
\end{aligned}
$$

leading to the derivatives

$$
\frac{\partial s_{\mathrm{f}}}{\partial \alpha_{\mu \nu}}=-\rho_{\mathrm{f}} H_{\alpha} \alpha^{\mu \nu}, \quad \frac{\partial s_{\mathrm{f}}}{\partial \hat{\omega}_{\mu}}=-\rho_{\mathrm{f}} H_{\omega} \hat{\omega}^{\mu},
$$

where $H_{\alpha}$ and $H_{\omega}$ must be positive to guarantee nonnegative entropy production. A quadratic entropy function is not only consistent with the second-order Israel-Stewart model and the standard formulation of EIT, but should also be sufficient for producing the linearized equations for our stability analysis.

\section{Energy-momentum tensor}

For the entropy function (14), the energy-momentum tensor (A3) becomes (see the Appendix)

$$
\begin{aligned}
T^{\mu \nu}= & \left(\rho_{\mathrm{f}} c^{2}+\epsilon_{\mathrm{f}}\right) u^{\mu} u^{v}+p_{\mathrm{f}} \hat{\eta}^{\mu \nu} \\
& +\rho_{\mathrm{f}} T_{\mathrm{f}}\left[2 H_{\alpha}\left(\alpha^{\mu \lambda} \alpha_{\lambda}{ }^{\nu}-\alpha^{\mu \nu}\right)+H_{\omega} \hat{\omega}^{\mu} \hat{\omega}^{\nu}\right] \\
& -\rho_{\mathrm{f}} T_{\mathrm{f}}^{2} H_{\omega}\left(\hat{\omega}^{\mu} u^{v}+u^{\mu} \hat{\omega}^{\nu}\right),
\end{aligned}
$$

where

$$
p_{\mathrm{f}}=T_{\mathrm{f}}\left(s_{\mathrm{f}}-\rho_{\mathrm{f}} \frac{\partial s_{\mathrm{f}}}{\partial \rho_{\mathrm{f}}}-\epsilon_{\mathrm{f}} \frac{\partial s_{\mathrm{f}}}{\partial \epsilon_{\mathrm{f}}}\right)
$$

is the pressure in the local rest frame. From a physical point of view, $u_{\mu} T^{\mu v} u_{v}=\rho_{\mathrm{f}} c^{2}+\epsilon_{\mathrm{f}}$ can be interpreted as the total internal energy density, with the first term on the right-hand side associated with the mass density and the second term associated with the thermodynamic state of the system. If the remaining terms on the right-hand side of Eq. (16) are contracted with either $u_{\mu}$ or $u_{v}$, only the term $\rho_{\mathrm{f}} T_{\mathrm{f}}^{2} H_{\omega}\left(\hat{\omega}^{\mu} u^{\nu}+\right.$ $u^{\mu} \hat{\omega}^{\nu}$ ) produces a nonzero result which, according to Eqs. (13) and (15), is given by the product of temperature and nonconvective entropy flux. This term has exactly the same structure as the terms associated with the heat and momentum fluxes in Ref. [17]. Finally, the contribution $p_{\mathrm{f}} \hat{\eta}^{\mu \nu}+$ $\rho_{\mathrm{f}} T_{\mathrm{f}}\left[2 H_{\alpha}\left(\alpha^{\mu \lambda} \alpha_{\lambda}{ }^{\nu}-\alpha^{\mu \nu}\right)+H_{\omega} \hat{\omega}^{\mu} \hat{\omega}^{\nu}\right]$ is the counterpart of the stress tensor in Ref. [17]. The term $\rho_{\mathrm{f}} T_{\mathrm{f}} H_{\omega} \hat{\omega}^{\mu} \hat{\omega}^{\nu}$ expresses a quadratic cross effect, relating heat fluxes and stresses. Note that only the core contribution $p_{\mathrm{f}} \hat{\eta}^{\mu \nu}-2 \rho_{\mathrm{f}} T_{\mathrm{f}} H_{\alpha} \alpha^{\mu \nu}$ contributes to the linearized stress tensor, so that we obtain a simple interpretation of the structural tensor $\alpha^{\mu \nu}$. As pointed out before, the dependence of the energy-momentum tensor on the form of the entropy function is a characteristic feature of nonequilibrium thermodynamics. The tensor $T^{\mu \nu}$ in Eq. (16) is manifestly symmetric.

\section{E. Model summary}

Summarizing, our model consists of 14 equations: the continuity equation (1), the energy-momentum tensor conservation equation (4), and the relaxation equations (5) and (9) for the structural variables $\omega_{\mu}$ and $\alpha_{\mu \nu}$, respectively. Assuming a quadratic entropy in the structural variables, the energy-momentum tensor is found to be of the form given in Eq. (16).

To close the model, two equations of state are required, to relate the variables $T_{\mathrm{f}}$ and $p_{\mathrm{f}}$ defined in Eqs. (12) and (17) to the independent thermodynamic variables $\epsilon_{\mathrm{f}}$ and $\rho_{\mathrm{f}}$. For the quadratic entropy density (14), $T_{\mathrm{f}}$ and $p_{\mathrm{f}}$ are fully determined by the function $s_{\mathrm{f}}^{\text {eq }}\left(\rho_{\mathrm{f}}, \epsilon_{\mathrm{f}}\right)$, so that there are only three independent second-order derivatives or thermodynamic material parameters. We use the isobaric thermal expansion coefficient $\alpha_{p}$, the adiabatic thermal expansivity $\alpha_{s}$ and the isothermal speed of sound $c_{T}$ as basic material properties 
(for a convenient summary of the relations between various thermodynamic material parameters, see Appendix A of Ref. [37]). Also the unknown variables are 14 in total: $\rho_{\mathrm{f}}, \epsilon_{\mathrm{f}}$, $u_{\mu}, \alpha_{\mu \nu}$ and $\omega_{\mu}(1+1+3+6+3=14)$, given the symmetry of the $\alpha_{\mu \nu}$ and the constraints in Eqs. (3), (8), and (11).

\section{LINEAR STABILITY ANALYSIS}

We now perform a linear stability analysis for the thermodynamically admissible model of relativistic fluid dynamics presented in the preceding section. Following standard procedures [10-12,17], we express the deviation $\delta q$ of any variable $q$ from its equilibrium value in the form $\delta q=\delta \tilde{q} e^{\Omega t+i k x^{1}}$, with $t$ the time and $x^{1}$ one of the space directions in the rest frame.

\section{A. Equilibrium rest frame}

We consider an equilibrium state corresponding to given (fixed) values of $\epsilon_{\mathrm{f}}, \rho_{\mathrm{f}}, T_{\mathrm{f}}$, and $p_{\mathrm{f}}$. The values of the velocity and the structural variables in such an equilibrium state are

$$
\begin{gathered}
u_{0}=-1, \quad u_{i}=0, \quad \hat{\eta}_{0 \mu}=\hat{\eta}_{\mu 0}=0, \quad \hat{\eta}_{i j}=\delta_{i j}, \\
\alpha_{00}=-1, \quad \alpha_{0 i}=\alpha_{i 0}=\alpha_{i j}=0, \quad \omega_{0}=T_{\mathrm{f}}, \quad \omega_{i}=0 .
\end{gathered}
$$

The full list of unknowns of the linearized problem is given by $\delta \rho, \delta T, \delta u_{1}, \delta u_{2}, \delta u_{3}, \delta \omega_{1}, \delta \omega_{2}, \delta \omega_{3}, \delta \alpha_{11}, \delta \alpha_{12}, \delta \alpha_{13}, \delta \alpha_{22}$, $\delta \alpha_{23}, \delta \alpha_{33}$. In view of the constraints (3), (8), and (11), the following relationships hold:

$$
\begin{gathered}
\delta u_{0}=0, \quad \delta \hat{\eta}_{00}=0, \quad \delta \hat{\eta}_{i 0}=\delta \hat{\eta}_{0 i}=\delta u_{i}, \\
\delta \alpha_{00}=0, \quad \delta \alpha_{0 i}=\delta \alpha_{i 0}=\delta u_{i}, \quad \delta \omega_{0}=\delta T .
\end{gathered}
$$

\section{B. Linearized equations}

To prepare the stability analysis, we write the linearized evolution equations of the model as

$$
\begin{gathered}
\partial_{0} \rho_{\mathrm{f}}=-\rho_{\mathrm{f}} \partial_{i} u_{i} \\
\partial_{0} \epsilon_{\mathrm{f}}=-\left(\epsilon_{\mathrm{f}}+p_{\mathrm{f}}-\rho_{\mathrm{f}} T_{\mathrm{f}}^{3} H_{\omega}\right) \partial_{i} u_{i}+\rho_{\mathrm{f}} T_{\mathrm{f}}^{2} H_{\omega} \partial_{i} \omega_{i} \\
\left(\rho_{\mathrm{f}} c^{2}+\epsilon_{\mathrm{f}}+p_{\mathrm{f}}-\rho_{\mathrm{f}} T_{\mathrm{f}}^{3} H_{\omega}\right) \partial_{0} u_{j} \\
=-\partial_{j} p_{\mathrm{f}}+2 \rho_{\mathrm{f}} T_{\mathrm{f}} H_{\alpha} \partial_{k} \alpha_{j k}+\rho_{\mathrm{f}} T_{\mathrm{f}}^{2} H_{\omega} \partial_{0} \omega_{j} \\
\partial_{0} \omega_{j}=\partial_{j} \omega_{0}-\frac{1}{c \lambda_{1}}\left(\omega_{j}+u_{j} \omega_{0}\right) \\
\partial_{0} \alpha_{j k}=\partial_{j} u_{k}+\partial_{k} u_{j}-\frac{1}{c \lambda_{2}}\left(\alpha_{j k}-\frac{\alpha_{l l}}{3} \delta_{j k}\right)-\frac{1}{c \lambda_{0}} \frac{\alpha_{l l}}{3} \delta_{j k}
\end{gathered}
$$

Moreover, it is useful for the further development to define the dimensionless parameters

$$
X=\frac{2 T_{\mathrm{f}} H_{\alpha}}{c_{T}^{2}}, \quad Y=\frac{T_{\mathrm{f}}^{2} H_{\omega}}{\alpha_{p} c_{T}^{2}}, \quad Z=T_{\mathrm{f}} \alpha_{p},
$$

where $c_{T}$ is the isothermal speed of sound and $\alpha_{p}$ the isobaric thermal expansivity. From Eqs. (20) and (21), we can derive an evolution equation for the temperature $T_{\mathrm{f}}\left(\rho_{\mathrm{f}}, \epsilon_{\mathrm{f}}\right)$,

$$
\partial_{0} T_{\mathrm{f}}=-\frac{1-Y}{\alpha_{s}} \partial_{i} u_{i}+\frac{Y}{T_{\mathrm{f}} \alpha_{s}} \partial_{i} \omega_{i}
$$

where we have used the formula $\hat{c}_{v}=T_{\mathrm{f}} \alpha_{p} \alpha_{s} c_{T}^{2}$ involving the specific heat at constant volume, $\hat{c}_{v}$, and the thermal expansivity at constant entropy, $\alpha_{s}$, as well as the formula (A.22) of Ref. [37] for $\partial T_{\mathrm{f}}\left(\rho_{\mathrm{f}}, \epsilon_{\mathrm{f}}\right) / \partial \rho_{\mathrm{f}}$. The equation of motion Eq. (22) can be rewritten as

$$
\partial_{0} u_{j}=Q\left(-\frac{1}{\rho_{\mathrm{f}}} \partial_{j} \rho_{\mathrm{f}}-\frac{Z}{T_{\mathrm{f}}} \partial_{j} T_{\mathrm{f}}+X \partial_{k} \alpha_{j k}+\frac{Y Z}{T_{\mathrm{f}}} \partial_{0} \omega_{j}\right),
$$

where we have used $\partial p_{\mathrm{f}}\left(\rho_{\mathrm{f}}, T_{\mathrm{f}}\right) / \partial T_{\mathrm{f}}=\rho_{\mathrm{f}} c_{T}^{2} \alpha_{p}$, which follows from $d \rho_{\mathrm{f}}=-\rho_{\mathrm{f}} \alpha_{p} d T_{\mathrm{f}}+\rho_{\mathrm{f}} \kappa_{T} d p_{\mathrm{f}}$ and $\rho_{\mathrm{f}} c_{T}^{2} \kappa_{T}=1$ [or see Eq. (A.33) of Ref. [37]], where $\kappa_{T}$ is the isothermal compressibility, and the definition

$$
Q=\frac{\rho_{\mathrm{f}} c_{T}^{2}}{\rho_{\mathrm{f}} c^{2}+\epsilon_{\mathrm{f}}+p_{\mathrm{f}}-Y Z \rho_{\mathrm{f}} c_{T}^{2}} .
$$

Natural ranges of parameters are $0<X, 0<Y<1,0<Q$, $0<\lambda_{j}$. The constraint on $X$ emerges from the definition of $X$ in (25), where all the terms are nonnegative. The constraint $0<Y$ is obvious from the definition, too. In nonrelativistic hydrodynamics, the sign of the temperature change associated with the relative volume change $c \partial_{i} u_{i}$ is opposite to the sign of $\alpha_{s}$ [see, for example, Eqs. (7.3) and (A.29) of Ref. [37]]; we hence assume that the factor $1-Y$ in Eq. (26) must always be positive, that is $Y<1$. From the physical point of view, $Q$ gives an indication on the relativistic character of a fluid. As the speed of sound $c_{T}$ approaches the speed of light $c$, the parameter $Q$ grows. One might be tempted to interpret $Q \rightarrow 0$ as the limit of a nonrelativistic fluid, however, Eq. (27) clearly shows that the nonrelativistic limit is much more subtle. The timescales $\lambda_{0}$ and $\lambda_{2}$ can be chosen independently, without the need to impose any further inequality, as it can be seen by splitting the tensorial relaxation equation (24) for the $\alpha_{j k}$ into its trace-free and trace parts:

$$
\begin{gathered}
\partial_{0} \stackrel{\circ}{\alpha}_{j k}=\partial_{j} u_{k}+\partial_{k} u_{j}-\frac{2}{3} \partial_{i} u_{i} \delta_{j k}-\frac{1}{c \lambda_{2}} \stackrel{\circ}{\alpha}_{j k}, \\
\partial_{0} \alpha_{i i}=2 \partial_{i} u_{i}-\frac{1}{c \lambda_{0}} \alpha_{i i} .
\end{gathered}
$$

\section{Block structure}

The linearized system can be written in the matrix form $\boldsymbol{M} \cdot \delta q=0$, where $\boldsymbol{M}$ is a $14 \times 14$ matrix with blockstructure $(6+3+3+1+1)$ and $\delta q=\left(\delta \rho, \delta T, \delta u_{1}, \delta \omega_{1}\right.$, $\delta \alpha_{11},\left(\delta \alpha_{22}+\delta \alpha_{33}\right) / 2, \delta u_{2}, \delta \omega_{2}, \delta \alpha_{12}, \delta u_{3}, \delta \omega_{3}, \delta \alpha_{13}, \delta \alpha_{22}-$ $\left.\delta \alpha_{33}, \delta \alpha_{23}\right)$. Without loss of generality, we can choose $\rho_{\mathrm{f}}=T_{\mathrm{f}}=c=\lambda_{1}=1$, which is equivalent to choosing the units for density, temperature, velocity and time. The matrix $\boldsymbol{M}$ is

$$
\boldsymbol{M}=\left[\begin{array}{lllll}
\boldsymbol{N} & 0 & 0 & 0 & 0 \\
0 & \boldsymbol{R} & 0 & 0 & 0 \\
0 & 0 & \boldsymbol{R} & 0 & 0 \\
0 & 0 & 0 & f & 0 \\
0 & 0 & 0 & 0 & f
\end{array}\right],
$$

with the blocks $\boldsymbol{N}=[6 \times 6], \boldsymbol{R}=[3 \times 3], f=[1 \times 1]$ on the diagonal, and the zeros indicating blocks of variable size 
containing only 0 elements. In particular,

$$
\begin{gathered}
\boldsymbol{R}=\left[\begin{array}{cccc}
\Phi & -Y Z \Phi & -X Q \mathrm{i} \\
3 G & \Phi+3 G & 0 \\
-\mathrm{i} & 0 & \Phi+3 F
\end{array}\right], \\
\boldsymbol{N}=\left[\begin{array}{ccccccc}
\Phi & 0 & \mathrm{i} & 0 & 0 & 0 \\
0 & \Phi & \frac{1-Y}{A} \mathrm{i} & -\frac{Y}{A} \mathrm{i} & 0 & 0 \\
Q \mathrm{i} & Q Z \mathrm{i} & \Phi & -Y Z Q \Phi & -X Q \mathrm{i} & 0 \\
0 & -\mathrm{i} & 3 G & \Phi+3 G & 0 & 0 \\
0 & 0 & -2 \mathrm{i} & 0 & \Phi+E+2 F & 2(E-F) \\
0 & 0 & 0 & 0 & E-F & \Phi+2 E+F
\end{array}\right] .
\end{gathered}
$$

In the above equations, we defined $\Phi=\Omega /(c k)$ as well as the additional dimensionless parameters

$$
A=\alpha_{s} T_{\mathrm{f}}, \quad E=\frac{1}{3 c k \lambda_{0}}, \quad F=\frac{1}{3 c k \lambda_{2}}, \quad G=\frac{1}{3 c k \lambda_{1}} .
$$

To assess the stability, we look for the roots $\Phi_{n}$ of the characteristic polynomial $\operatorname{det} \boldsymbol{M}=0$, with $n=1, \ldots, 14$, i.e., we look for the values $\Phi_{n}$ for which the system has nontrivial solutions $\delta \tilde{q}_{n}$. Note that this is not a standard eigenvalue problem because $\Phi$ occurs also in one of the off-diagonal matrix elements of $\boldsymbol{R}$ and of $\boldsymbol{N}$ (we hence refer to $\Phi_{n}$ and $\Omega_{n}=c k \Phi_{n}$ as roots rather than eigenvalues). We assume $k>0$, so that initial perturbations $\delta \tilde{q}_{n}$ will decay in time and the system will be stable provided that $\operatorname{Re}\left(\Omega_{n}\right)<0$ for all $n$ $\left(\operatorname{Re}\left(\Omega_{n}\right)=0\right.$ corresponds to marginal stability of mode $\left.n\right)$.

Given the block structure of the matrix $\boldsymbol{M}$ the characteristic polynomial can be factorized as

$$
\operatorname{det} \boldsymbol{M}=\operatorname{det} \boldsymbol{N} \cdot(\operatorname{det} \boldsymbol{R})^{2} \cdot f^{2}=0 .
$$

We consider the different blocks separately. The solutions of the characteristic polynomial (36) are found for

$$
\begin{gathered}
f=\Phi+3 F=0, \\
\operatorname{det} \boldsymbol{R}=3 G Q X+\Phi(9 F G+Q X+9 F G Y Z) \\
+\Phi^{2}(3 F+3 G+3 G Y Z)+\Phi^{3}=0, \\
\operatorname{det} \boldsymbol{N}=0 .
\end{gathered}
$$

Equation (37) implies $\Phi=-3 F$, which is always real and negative, thus corresponding to decaying perturbations. This solution corresponds to $\Omega=-1 / \lambda_{2}$, i.e., a constant decay rate related to the characteristic relaxation rate of $\stackrel{\circ}{\alpha \nu}_{\mu}$ and independent of $k$, the spatial wavelength of the perturbation. This condition characterizes the independent relaxation of $\delta \alpha_{22}-\delta \alpha_{33}$ and $\delta \alpha_{23}$.

In the physically acceptable range of parameters, Eq. (38), which is associated with shear and transverse mass and heat fluxes $\left(\delta u_{2}, \delta \omega_{2}, \delta \alpha_{12}\right.$ or $\left.\delta u_{3}, \delta \omega_{3}, \delta \alpha_{13}\right)$, has only solutions with negative real parts. To proof this, we apply the Routh-Hurwiz criterion [38] for a third-order polynomial $a_{0}+a_{1} \Phi+a_{2} \Phi^{2}+\Phi^{3}$. According to this criterion, such a polynomial has only solutions with a negative real part if $a_{i}>0$ for all $i$ and $a_{1} a_{2}>a_{0}$. The first condition can be verified from Eq. (38), given the physically acceptable ranges of the parameters, which must all be real and positive. The second condition can be rewritten as

$$
\begin{aligned}
a_{2} a_{1}-a_{0}= & 27 F G\left(F+G+F Y Z+3 G Y Z+G Y^{2} Z^{2}\right) \\
& +3 Q X(F+G Y Z)>0,
\end{aligned}
$$

which is true for real valued, positive $F, G, X, Y, Z$. In the limit case $Q=0$, one solution of Eq. (38) is the purely real negative $\Phi=-3 F$ associated with the relaxation of $\alpha_{12}$ or $\alpha_{13}$ in the absence of transverse mass or heat flux. Additional roots of Eq. (38) for $Q=0$ are $\Phi=-3 G(1+Y Z)$ and $\Phi=$ 0 . The latter corresponds to time-independent perturbations of marginal stability. The mode associated with $\Phi=-3 G(1+$ $Y Z$ ) must satisfy the condition $\delta u_{2}+Y Z \delta \omega_{2}=0$ (or, $\delta u_{3}+$ $Y Z \delta \omega_{3}=0$ ); this relaxation mode exists for small positive $Q$, but does not exist for $Q=0$, because Eq. (27) then implies $\delta u_{2}=\delta u_{3}=0$.

We now consider Eq. (39). To determine the sign of the roots, we rearrange Eq. (39) as

$$
X=-\frac{(3 E+\Phi)(3 F+\Phi)\left\{\left(\Phi^{2}+Q\right)[A \Phi(3 G+\Phi)+Y]+\Phi Q\left[\Phi(-1+Y)^{2}+3 G\left(1+A \Phi^{2} Y\right)\right] Z\right\}}{2 \Phi(2 E+F+\Phi) Q[A \Phi(3 G+\Phi)+Y]}
$$

which can be compactly rewritten as $X=-N / D$, with $N$ the numerator and $D$ the denominator of the fraction (41). In the case of $\Phi$ purely real, positive values of $\Phi$ would lead to both $N>0$ and $D>0$, hence $X<0$. Since these are physically unacceptable values of $X$, purely real positive roots are not possible and purely real roots $\Phi$ can only be negative. Purely imaginary values of $\Phi$ correspond to an oscillatory nongrowing solution of the linear system and and imply marginal stability. 

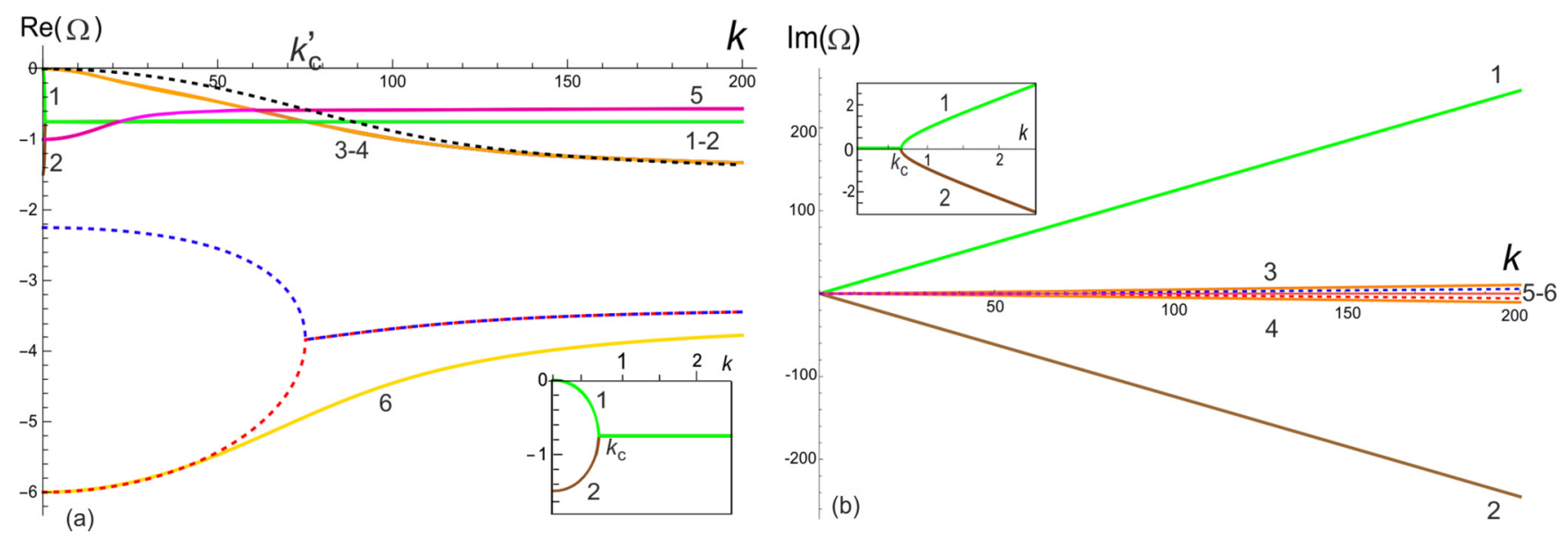

FIG. 1. (a) Real and (b) imaginary part of the roots $\Omega$ as a function of $k$, for $Q=0.001$. Other parameters are $A=1 / 3, X=1, Y=1 / 2$, $Z=1, \lambda_{0}=1, \lambda_{1}=2 / 3$, and $\lambda_{2}=1 / 6$. The dashed lines represent the roots of the characteristic polynomial of the $3 \times 3$ block $R$; the solid lines represent the roots of the characteristic polynomial of the $6 \times 6$ block $N$. Both $\Omega$ and $k$ are depicted in nondimensional units.

Therefore further investigation is required only for the case of a complex-valued root,

$$
\Phi=R+\mathrm{i} I
$$

with $R=\operatorname{Re}(\Phi) \neq 0$ and $I=\operatorname{Im}(\Phi) \neq 0$. We substitute Eq. (42) into Eq. (41), and we impose $\operatorname{Im}(X)=0$, since we know from Eq. (25) that $X$ has to be a real number. This condition is expressed as $Q=Q(R, I, E, F, G, Y, Z)$ and inserted into Eq. (41), thus finding

$$
X=-\frac{\left[I^{2}+(3 E+R)^{2}\right]\left[I^{2}+(3 F+R)^{2}\right] T_{1}}{\left.2|\Phi|^{2}\left\{6 E^{2}(3 F+2 R)+2\left(F I^{2}+3 F^{2} R+R|\Phi|^{2}+3 F R^{2}\right)+E I^{2}+9(F+R)^{2}\right]\right\} T_{2}},
$$

with

$$
\begin{aligned}
T_{1}= & 2 A R\left\{A\left[2 I^{2} R^{2}+R^{4}+3 G(2 R+3 G)|\Phi|^{2}\right]+2 R(3 G+R) Y\right\}+2 R\left(A I^{2}-Y\right)^{2} \\
& +3 G Z|\Phi|^{2}\left[2 A R(3 G+2 R)+Y\left(A|\Phi|^{2}-1\right)^{2}\right]+Z|\Phi|^{2}\left[2 A R|\Phi|^{2}(1-Y)^{2}\right] \\
T_{2}= & \left(A I^{2}-Y\right)^{2}+A\left\{A\left[2 I^{2} R^{2}+R^{4}+3 G|\Phi|^{2}(3 G+2 R)\right]+2 R(3 G+R) Y\right\}
\end{aligned}
$$

We compactly rewrite Eq. (43) as $X=-N^{\prime} / D^{\prime}$, where $N^{\prime}$ and $D^{\prime}$ are the numerator and the denominator of the fraction, respectively. From the equations above, one can see that $T_{1}>0$ and $T_{2}>0$, in the case of $R>0$. Thus $N^{\prime}>0$, $D^{\prime}>0$ and $X<0$, which is at variance with the physically acceptable values, $X>0$. Therefore, we have established $R=\operatorname{Re}(\Phi) \leqslant 0$ and completed the proof of the unconditional stability of the system, within the physically acceptable range of values of the parameters.

In Figs. 1-3 we show the real and imaginary parts of $\Omega$ as a function of $k$ for different values of the parameter $Q: Q=$ $0.001, Q=0.1$, and $Q=1$. The other parameters are given by $X=1, Y=1 / 2, Z=1, A=1 / 3, \lambda_{0}=1, \lambda_{1}=2 / 3$, and $\lambda_{2}=1 / 6$. The solid lines represent the roots derived from the $6 \times 6$ block $N$ [see Eq. (39)]. The dashed lines represent the roots derived from the $3 \times 3$ block $\boldsymbol{R}$ [see Eq. (38)]. Since there are two identical $\boldsymbol{R}$ blocks in the matrix $\boldsymbol{M}$, the multiplicity of each dashed line is two. The colors (online) in the figures are redundant with the numbering, to facilitate the readability.

In the case of $Q=0.001$, we find several modes that have been previously identified by other authors [10]: the line labeled 1 (green online) is characterized by a proportionality to $-k^{2}$ near $k=0$ and identifies a diffusive mode for $k<k_{c}$, where $k_{c}$ is a characteristic wavelength. The line 2 (brown online), represents relaxation of the heat flux $\omega_{1}$ : this can be seen from the fact that, for $k=0$, it takes the value $\Omega=-1 / \lambda_{1}$ [see Eq. (23)]. The real parts of 1 and 2 merge for $k>k_{c}$, while the complex parts becomes nonzero and conjugate, turning both 1 and 2 into two propagating nonhydrodynamic modes. The roots 3 and 4 (orange online) are complex conjugate with negative real part, hence they represent two sound modes. The roots 5 (magenta online) and 6 (yellow online) have negative real part, reaching an asymptote as $k$ increases, and vanishing complex part, thus they can be identified as two nonpropagating relaxation modes. In particular, at $k=0$, root 5 takes the value $\Omega=-1 / \lambda_{0}$ while root 6 takes the value $\Omega=-1 / \lambda_{2}$. Hence we conclude that the mode 5 is related to the relaxation of $\alpha_{i i}$ [see Eq. (30)] and the mode 6 is related to the relaxation of $\stackrel{\alpha}{\alpha k}_{j k}$ [see Eq. (29)]. From the $3 \times 3$ block $\boldsymbol{R}$, three modes appear, represented by the dashed lines: for small $k$, one mode represents relaxation of $\alpha_{12}$ (or $\left.\alpha_{13}\right)$ in the absence of transverse mass or heat fluxes (light-gray, or red online) the other one represents relaxation of $\omega_{2}$ (or $\left.\omega_{3}\right)$ in 


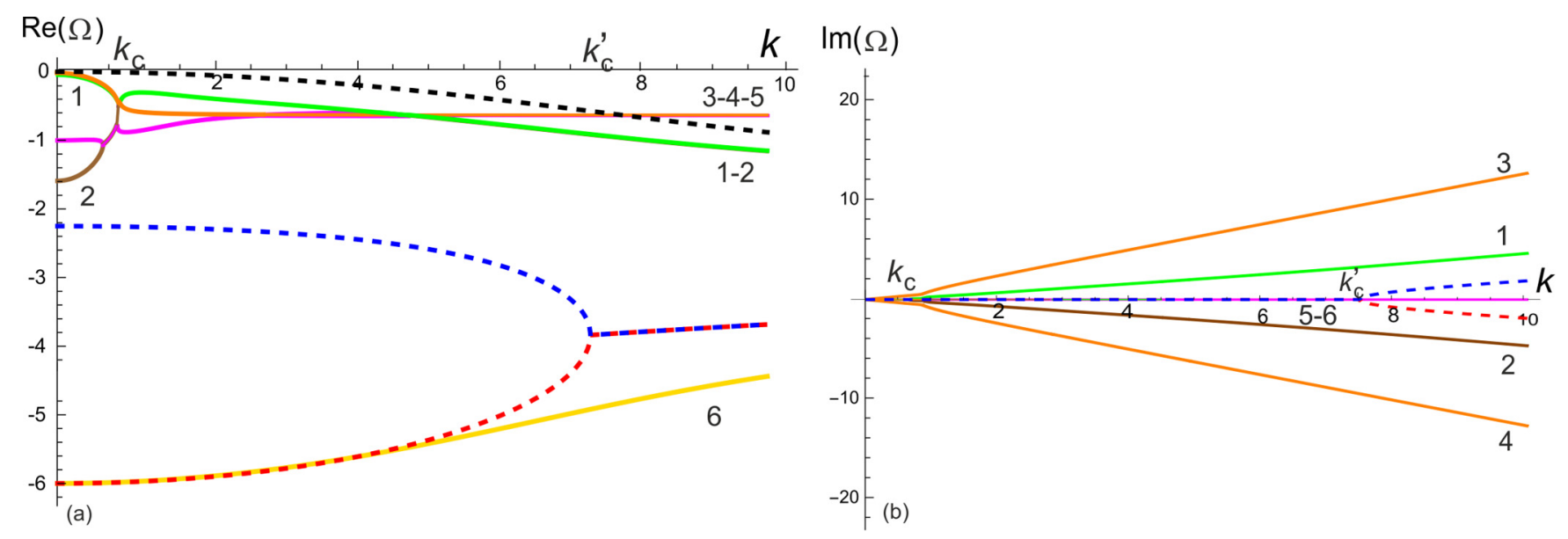

FIG. 2. (a) Real and (b) imaginary part of the roots $\Omega$ as a function of $k$, for $Q=0.1$. For other parameters and line style code see Fig. 1 .

the presence of transverse mass and momentum fluxes (darkgray, or blue online); these two modes turn into propagating modes for $k$ larger than a characteristic value $k_{c}^{\prime}$. The third mode displays diffusive $-k^{2}$ behavior at small $k$ and saturates at large $k$ (black). For $Q=1$, the roots 1 and 2 are shifted down from the origin, while all other modes retain a similar meaning. In the case of $Q=0.1$, an intermediate situation between the two previous cases appears and the different modes are combined in a nontrivial way.

\section{SUMMARY AND DISCUSSION}

In the present paper we considered the thermodynamically consistent model for relativistic fluid dynamics developed within the GENERIC framework [29,30], where the treatment of nonequilibrium phenomena (momentum and heat transport) is described by means of additional structural variables to prevent the problem of infinite propagation speeds. We performed a linear stability analysis of the equilibrium rest state and we analytically proved that, in the entire range of the physically meaningful parameters, the model is unconditionally stable.

In nonrelativistic fluid dynamics, the equations for liquids and gases possess the same Navier-Stokes-Fourier form and differ only by the characteristic values of the transport coefficients. This is a consequence of the fact that the basic equations express the balance laws for the conserved quantities mass, momentum and energy in a straightforward form. In relativistic fluid dynamics, we are faced with additional relaxation equations for nonconserved structural variables so that there is no reason to expect universal equations that encompass both liquids and gases.

For a gas described by the Boltzmann equation, all macroscopic properties are strongly related to the velocities of the particles. For a liquid, the interactions between particles and the energy stored in the bonds are more relevant than the velocities themselves. Our equations of relativistic fluid dynamics include a Poisson-bracket formulation of the convection mechanism. A Poisson-bracket formulation has not been found for the 13- or 14-moment equations derived from the nonrelativistic or relativistic Boltzmann equations. The problem is that the convection terms for increasingly higher moments in momentum space are strongly coupled and hence very complicated so that they become difficult, if not impossible, to truncate. The situation is very different if the structural variables describe features related to forces and differences in position space. This is the reason why we expect a serious difference between fluid dynamics of
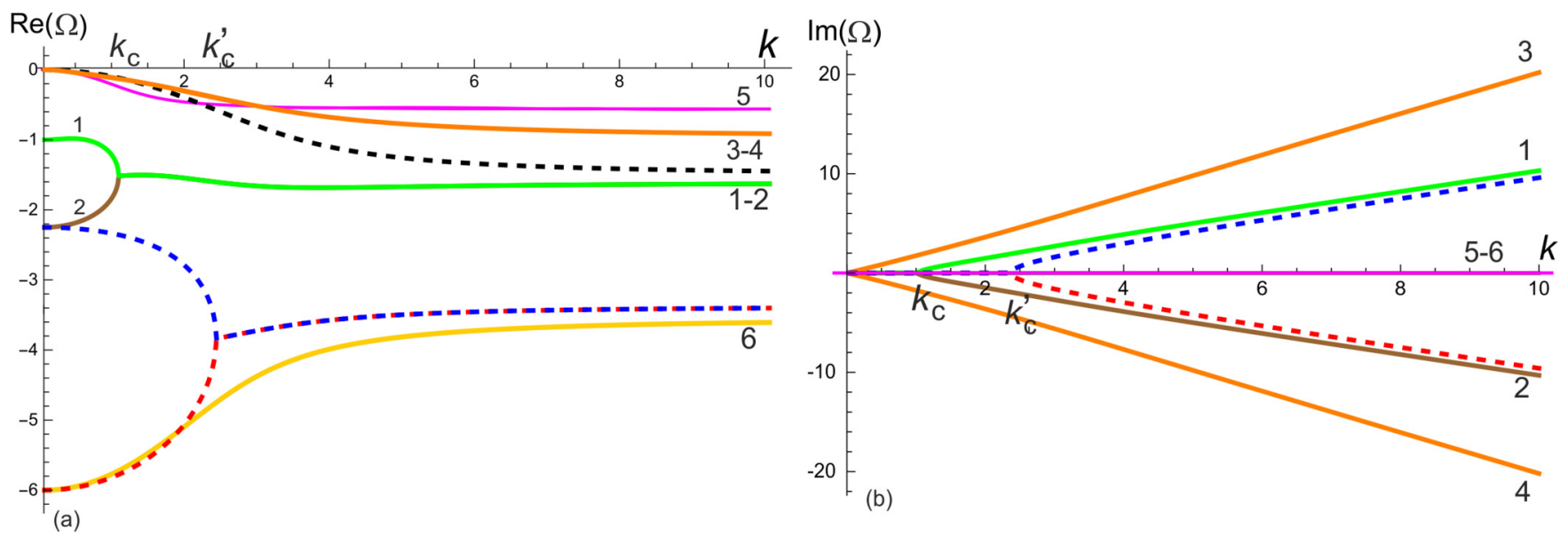

FIG. 3. (a) Real and (b) imaginary part of the roots $\Omega$ as a function of $k$, for $Q=1$. For other parameters and line style code see Fig. 1 . 
relativistic liquids and gases. The heuristic derivation of the GENERIC equations based on the knowledge of complex fluids in Ref. [37] underlines their liquidlike background, whereas the Israel-Stewart model and the EIT equations are based on moment expansions for the Boltzmann equation and hence are appropriate for gaslike fluids. Note that, in the case of quark-gluon plasmas, recent experimental findings showed that a liquidlike description may be more accurate [39]. For gaslike fluids, a Poisson-bracket formulation of the convection mechanism has so far been found only in the Boltzmann equation or the equivalent infinite moment hierarchy.

With the present paper we performed one step forward in showing that the thermodynamically consistent model developed within the GENERIC framework [29,30] should be considered as a valuable option, a worthwhile alternative to the existing ones, when addressing the study of relativistic fluids. To gain full confidence, linear stability should also be investigated in a boosted system [10], which is considerably more complicated and hence beyond the scope of the present analytical study (more terms in evolution equations, angle between boost and wave vector of perturbation). The elegance associated with the Poissonian structure of the convection terms in the relaxation equations (5) and (9) for the structural variables is an important argument in favor of the equations derived within the GENERIC framework. More physical entropy functions, presumably containing logarithmic rather than quadratic contributions, open the door to more realistic modeling, where the general form (A3) of the energy-momentum tensor is prescribed by thermodynamic admissibility.

\section{ACKNOWLEDGMENTS}

The authors would like to express their particular gratitude to Martin Kröger, who greatly contributed to the final proof of the stability of the problem. They would also like to thank Alberto Montefusco, Martin Callies, and Michele Graf for insightful discussions.

\section{APPENDIX: NOTATIONAL CORRESPONDENTS}

The original formulation of GENERIC equations for relativistic fluid dynamics in Sec. 5.2 of the monograph [36] and Sec. 2 of the paper [29] is based on the inverse function $\epsilon_{\mathrm{f}}\left(\rho_{\mathrm{f}}, s_{\mathrm{f}}, \alpha_{\mu \nu}, \hat{\omega}_{\mu}\right)$ rather than $s_{\mathrm{f}}\left(\rho_{\mathrm{f}}, \epsilon_{\mathrm{f}}, \alpha_{\mu \nu}, \hat{\omega}_{\mu}\right)$. Moreover, in the original work, six independent scalar invariants are formed from $\alpha_{\mu \nu}$ and $\hat{\omega}_{\mu}$ and used as the explicit arguments of $\epsilon_{\mathrm{f}}$. In Ref. [36], all partial derivatives of $\epsilon_{\mathrm{f}}$ are contained in Eq. (5.76). The relevant notational correspondents required for translating the results of Ref. [36] or [29] for the entropy balance and the energy-momentum tensor into the present notation are compiled in Table I.

The entropy balance equation (13) can be obtained from Eqs. (5.49), (5.84), and (5.102) of [36] or from Eqs. (27), (32),
TABLE I. Notational correspondents.

\begin{tabular}{lcc}
\hline \hline This paper & Ref. [36] & Ref. [29] \\
\hline$T_{\mathrm{f}}$ & $T_{\mathrm{f}}^{*}$ & $T_{\mathrm{f}}^{*}$ \\
$\lambda_{j}$ & $\tau_{j}$ & $\tau_{j}$ \\
$\alpha_{\mu \nu}$ & $c_{\mu \nu}$ & $c_{\mu \nu}$ \\
$\omega_{\mu}$ & $-c w_{\mu}$ & $-c w_{\mu}$ \\
$\frac{\partial s_{\mathrm{f}}}{\partial \alpha_{\mu \nu}}$ & $-\frac{1}{2 T_{\mathrm{f}}^{*}} \phi^{* \mu v}$ & $-\frac{1}{2 T_{\mathrm{f}}^{*}} \phi^{\mu v}$ \\
$\frac{\partial s_{\mathrm{f}}}{\partial \hat{\omega}_{\mu}}$ & $\frac{1}{c T_{\mathrm{f}}^{*}} \hat{\eta}^{\mu v} \sigma_{v v^{\prime}}^{*} w^{v^{\prime}}$ & $\frac{1}{c T_{\mathrm{f}}^{*}}\left(\eta^{\mu \nu}+u^{\mu} u^{v}\right) \sigma_{v v^{\prime}} w^{v^{\prime}}$ \\
\hline \hline
\end{tabular}

and (33) of Ref. [29]. According to Eqs. (5.55) and (5.86) of Ref. [36] [or, equivalently, Eqs. (21) and (30) of Ref. [29]], the energy-momentum tensor is given by

$$
\begin{aligned}
T^{\mu \nu}= & \left(\rho_{\mathrm{f}} c^{2}+\epsilon_{\mathrm{f}}+T_{\mathrm{f}}^{*} \varsigma\right) u^{\mu} u^{\nu}+p_{\mathrm{f}} \hat{\eta}^{\mu \nu} \\
& +\phi^{* \mu \lambda}\left(c_{\lambda}{ }^{\nu}-\eta_{\lambda}{ }^{\nu}\right)+\sigma^{* \mu \lambda} w_{\lambda} w^{\nu}
\end{aligned}
$$

with $\varsigma=u_{\mu} \sigma^{* \mu v} w_{\nu} / c$. The symmetry of $T^{\mu \nu}$ is proven in Exercise 104 of Ref. [36] or displayed explicitly in Eq. (34) of Ref. [29]; the proof makes explicit use of the fact that $\epsilon_{\mathrm{f}}$ depends only on scalar invariants. From Table I, we find

$$
\sigma^{* \mu \lambda} w_{\lambda}=c T_{\mathrm{f}}^{*} \frac{\partial s_{\mathrm{f}}}{\partial \hat{\omega}_{\mu}}-c \varsigma u^{\mu},
$$

so that, in the notation of the present paper, we obtain

$$
\begin{aligned}
T^{\mu \nu}= & \left(\rho_{\mathrm{f}} c^{2}+\epsilon_{\mathrm{f}}\right) u^{\mu} u^{v}+p_{\mathrm{f}} \hat{\eta}^{\mu \nu}-2 T_{\mathrm{f}} \frac{\partial s_{\mathrm{f}}}{\partial \alpha_{\mu \lambda}}\left(\alpha_{\lambda}^{v}-\eta_{\lambda}^{v}\right) \\
& -T_{\mathrm{f}} \frac{\partial s_{\mathrm{f}}}{\partial \hat{\omega}_{\mu}} \hat{\omega}^{v}+T_{\mathrm{f}}^{2} \frac{\partial s_{\mathrm{f}}}{\partial \hat{\omega}_{\mu}} u^{v}+\varsigma u^{\mu} \hat{\omega}^{v} .
\end{aligned}
$$

In practice, $\varsigma$ can be regarded as a fitting parameter for making $T^{\mu \nu}$ symmetric. For the quadratic entropy density (14), we have

$$
T_{\mathrm{f}}^{2} \frac{\partial s_{\mathrm{f}}}{\partial \hat{\omega}_{v}}=\varsigma \hat{\omega}^{v},
$$

so that we find the energy-momentum tensor (16).

A simple heuristic derivation of the GENERIC equations of relativistic fluid dynamics has been attempted in Sec. 12.6 of the textbook [37]. The key ingredients are a convection mechanism based on the upper-convected derivatives used in modeling complex fluids with tensor and vector variables and the formulation of a proper entropy balance equation. For quadratic entropy density, which is the recommended choice for linear relaxation, the GENERIC results (A3), (A4) have been fully reproduced. For more general entropy densities, the last term in Eq. (A3) (involving 5 ) is not reproduced correctly by the last term of Eq. (12.86) of Ref. [37]. This problem occurs when the symmetry of $T^{\mu \nu}$ becomes a subtle issue because $\partial s_{\mathrm{f}} / \partial \alpha_{\mu \lambda}$ and $\alpha_{\lambda}{ }^{\nu}$ do not commute.
[1] L. D. Landau, On the multiple production of particles during collisions of fast particles, Izv. AN SSSR Ser. Fiz. 17, 51 (1953).

[2] I.M. Khalatnikov, Some questions of the relativistic hydrodynamics, J. Exp. Theor. Phys. 27, 529 (1954).
[3] J. Adams et al. (STAR Collaboration), Experimental and theoretical challenges in the search for the quark-gluon plasma: The STAR Collaboration's critical assessment of the evidence from RHIC collisions, Nucl. Phys. A 757, 102 (2005). 
[4] K. Adcox et al. (PHENIX Collaboration), Formation of dense partonic matter in relativistic nucleus-nucleus collisions at RHIC: Experimental evaluation by the PHENIX Collaboration, Nucl. Phys. A 757, 184 (2005).

[5] I. Arsene et al. (BRAHMS Collaboration), Quark-gluon plasma and color glass condensate at RHIC? The perspective from the BRAHMS experiment, Nucl. Phys. A 757, 1 (2005).

[6] B. B. Back et al. (PHOBOS Collaboration), The PHOBOS perspective on discoveries at RHIC, Nucl. Phys. A 757, 28 (2005).

[7] C. Eckart, The thermodynamics of irreversible processes. III. Relativistic theory of the simple fluid, Phys. Rev. 58, 919 (1940).

[8] L. Landau and E. M. Lishiftz, in Fluid Mechanics (Addison Wesley, Reading, 1958), p. 500.

[9] G. S. Denicol, T. Kodama, T. Koide, and P. Mota, Stability and causality in relativistic dissipative hydrodynamics, J. Phys. G 35, 115102 (2008).

[10] S. Pu, T. Koide, and D. H. Rischke, Does stability of relativistic dissipative fluid dynamics imply causality? Phys. Rev. D 81, 114039 (2010).

[11] W. A. Hiscock and L. Lindblom, Stability and causality in dissipative relativistic fluids, Ann. Phys. 151, 466 (1983).

[12] W. A. Hiscock and L. Lindblom, Generic instabilities in firstorder dissipative relativistic fluid theories, Phys. Rev. D 31, 725 (1985).

[13] N. G. van Kampen, A model for relativistic heat transport, Physica 46, 315 (1970).

[14] C. Cattaneo, Sur une forme de l'équation de la chaleur éliminant le paradoxe d'une propagation instantanée, Comp. Rend. Acad. Sci. Paris 247, 431 (1958).

[15] M. Kranyš, Relativistic hydrodynamics with irreversible thermodynamics without the paradox oi infinite velocity of heat conduction, Nuovo Cimento B 42, 51 (1966).

[16] P. Kostädt and M. Liu, On the causality and stability of the relativistic diffusion equation, Phys. Rev. D 62, 023003 (2000).

[17] P. Ván and T. S. Biró, Relativistic hydrodynamics-Causality and stability, Eur. Phys. J. Spec. Top. 155, 201 (2008).

[18] I. Müller, Zum Paradoxon der Wärmeleitungstheorie, Z. Phys. 198, 329 (1967).

[19] H. Grad, On the kinetic theory of rarefied gases, Commun. Pure Appl. Math. 2, 331 (1949).

[20] J. M. Stewart, in Non-Equilibrium Relativistic Kinetic Theory (Springer-Verlag, Berlin, 1971), pp. 78-81.

[21] J. L. Anderson, in Relativity, edited by M. Carmeli, S. I. Fickler, and L. Witten (Springer, Boston, MA, 1971), p. 109.
[22] C. Marle, Sur l' établissement des équations de l'hydrodynamique des fluides relativistes dissipatifs, Ann. Inst. H. Poincaré A 10, 67 (1969).

[23] M. Kranyš, Non truncated relativistic Chernikov-Grad 13moment equations as an approach to nonstationary thermodynamics, Phys. Lett. A 33, 77 (1970).

[24] W. Israel, Nonstationary irreversible thermodynamics: A causal relativistic theory, Ann. Phys. 100, 310 (1976).

[25] W. Israel and J. M. Stewart, On transient relativistic thermodynamics and kinetic theory. II, Proc. Roy. Soc. Lond. A 365, 43 (1979).

[26] H. C. Öttinger, On the stupendous beauty of closure, J. Rheol. 53, 1285 (2009).

[27] D. Jou, J. Casas-Vazquez, and G. Lebon, Extended irreversible thermodynamics, Rep. Prog. Phys. 51, 1105 (1988).

[28] B. Betz, D. Henkel, and D. H. Rischke, From kinetic theory to dissipative fluid dynamics, Prog. Part. Nucl. Phys. 62, 556 (2009).

[29] H. C. Öttinger, Relativistic and nonrelativistic description of fluids with anisotropic heat conduction, Physica A 254, 433 (1998).

[30] H. C. Öttinger, On the structural compatibility of a general formulation for nonequilibrium dynamics with special relativity, Physica A 259, 24 (1998).

[31] H. C. Öttinger, Thermodynamically admissible equations for causal dissipative cosmology, galaxy formation, and transport processes in a gravitational collapse, Phys. Rev. D 60, 103507 (1999).

[32] P. Ilg and H. C. Öttinger, Nonequilibrium relativistic thermodynamics in bulk viscous cosmology, Phys. Rev. D 61, 023510 (1999).

[33] B. Carter, Convective variational approach to relativistic thermodynamics of dissipative fluids, Proc. Roy. Soc. Lon. A 433, 45 (1991).

[34] R. Baier, P. Romatschke, D. T. Son, A. O. Starinets, and M. A. Stephanov, Relativistic viscous hydrodynamics, conformal invariance, and holography, J. High Energy Phys. 04 (2008) 100.

[35] A. N. Beris and B. J. Edwards, The Thermodynamics of Flowing Systems (Oxford University Press, New York, 1994).

[36] H. C. Öttinger, in Beyond Equilibrium Thermodynamics (John Wiley \& Sons, Hoboken, NJ, 2005), p. 436.

[37] D. C. Venerus and H. C. Öttinger, A Modern Course in Transport Phenomena (Cambridge University Press, Cambridge, 2018).

[38] A. Hurwitz, Über die Bedingungen, unter welchen eine Gleichung nur Wurzeln mit negativen reellen Theilen besitzt, Math. Ann. 46, 273 (1895).

[39] The STAR Collaboration, Global $\Lambda$ hyperon polarization in nuclear collisions, Nature (London) 548, 62 (2017). 